\title{
Avaliação da capacidade de decisão de idosos diagnosticados com depressão maior
}

\author{
Assessment of the decision-making capacity of elderly individuals diagnosed \\ with major depression
}

\author{
Anelise Crippa ${ }^{1} \bowtie$, Irenio Gomes ${ }^{2}$, Newton Luiz Terra ${ }^{2}$ \\ ${ }^{1}$ Faculdade de Direito do Centro Universitário Cenecista de Osório (UNICNEC). Osório, RS. \\ 2 Escola de Medicina da Pontifícia Universidade Católica do Rio Grande do Sul (PUCRS). Porto Alegre, RS.
}

\section{RESUMO}

OBJETIVOS: Verificar se há alteração na capacidade de decisão em idosos com depressão maior.

MÉTODOS: Foi realizado um estudo de coorte prospectiva com análise transversal inicial, no período de janeiro de 2014 a setembro de 2015. Para o grupo de estudo foram selecionados pacientes idosos do Ambulatório de Envelhecimento Cerebral do Hospital São Lucas da PUCRS, em Porto Alegre, RS, diagnosticados com depressão maior atual e ainda sem tratamento para esse transtorno. Um grupo controle foi composto por idosos da mesma comunidade, sem depressão ou problemas cognitivos. Para avaliação da capacidade de decisão foi utilizada a Escala de Avaliação de Capacidade de Decisão (ESCADE), desenvolvida e validada em uma etapa anterior da pesquisa. Esse instrumento dividide-se em quatro domínios: atividade diária, gestão financeira, autogestão e bem-estar. A Escala de Depressão Geriátrica, forma abreviada, e o instrumento Mini International Neuropsychiatric Interview, versão detalhada, foram utilizados para avaliar a presença de depressão. O Instrumento de Triagem para Demência Vellore e o Exame Cognitivo de Addenbrooke versão revisada foram aplicados para avaliar declínio cognitivo. A capacidade de decisão foi comparada entre os dois grupos de idosos. A mesma avaliação foi feita nos idosos com depressão após seis meses de tratamento psiquiátrico. A análise estatística incluiu os testes t de Student, qui-quadrado de Pearson, Mann-Whitney e Wilcoxon. Foi considerado significativo um $\mathrm{p} \leq 0,05$.

RESULTADOS: Participaram da pesquisa 48 idosos com depressão maior e 144 idosos no grupo controle. A pontuação média da ESCADE

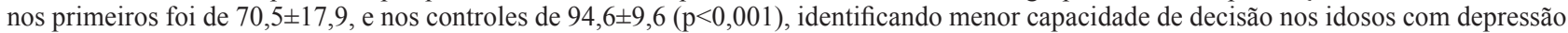
maior. Os domínios com maior diferença nas médias de pontuação foram o de autogestão (deprimidos $65,0 \pm 23,3$ e controles $97,8 \pm 6,2$ ) e o

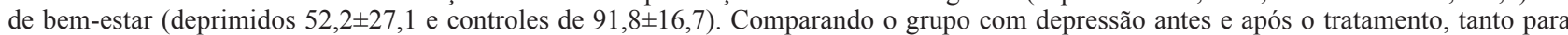
a pontuação geral quanto para cada um dos quatro domínios da ESCADE, a capacidade de tomada de decisão aumentou após o tratamento. CONCLUSÕES: O grupo de idosos com depressão maior atual apresentou menor capacidade de decisão em relação ao grupo controle. Houve melhora na capacidade de decisão após seis meses de tratamento psiquiátrico.

DESCRITORES: transtorno depressivo maior; idoso; autonomia; geriatria; gerontologia.

\section{ABSTRACT}

AIMS: To verify if there are changes in the decision-making capacity of elderly patients with major depression.

METHODS: This is a prospective cohort study with an initial cross-sectional analysis conducted between January 2014 and September 2015. Treatment-naïve elderly patients from the Cerebral Aging Outpatient Clinic at PUCRS São Lucas Hospital, in Porto Alegre, Brazil, all diagnosed with major depressive disorder, were selected. The control group was composed of elderly individuals from the same community who had neither depression nor cognitive problems. For evaluation of the decision-making capacity, the Assessment Scale for Decision-Making Capacity was designed and validated in a previous stage of the research. This instrument is divided into four domains: daily activity, financial management, self-management, and well-being. The Geriatric Depression Scale (short version) and the Mini-International Neuropsychiatric Interview (detailed version) were used to evaluate the presence of depression. The Vellore Screening Instrument for Dementia and the Addenbrooke's Cognitive Examination (revised version) were applied to evaluate cognitive decline. Decision-making capacity was compared between the two groups of elderly individuals. The same evaluation was conducted with elderly patients with depression after six months of psychiatric treatment. The statistical analysis included Student's t, Pearson's chi-square, Mann-Whitney, and Wilcoxon tests. Statistical significance was set at $\mathrm{p} \leq 0.05$.

RESULTS: Forty-eight elderly patients with major depression and 144 elderly individuals from the control group participated in the study. The Assessment Scale for Decision-Making Capacity average score in depression patients was $70.5 \pm 17.9$, compared to $94.6 \pm 9.6$ ( $p<0.001$ ) in the control group, which indicates poorer decision-making capacity among patients with major depression. The domains with the most striking differences in the average scores were self-management (depression patients $65.0 \pm 23.3$ and control group 97.8 \pm 6.2 ) and well-being (depression patients 52.2 \pm 27.1 and control group 91.8 \pm 16.7 ). When comparing patients with depression before and after treatment, regarding both the general score and the four domains of the Assessment Scale for Decision-Making Capacity, the decision-making capacity was higher after the treatment.

CONCLUSIONS: The group of elderly patients with current major depression had a lower decision-making capacity compared to the control group. Their decision-making capacity improved after six months of psychiatric treatment.

KEY WORDS: major depressive disorder; elderly; autonomy; geriatrics; gerontology. 
Abreviaturas: ACS, Agente Comunitário de Saúde; AMBEC, Ambulatório de Envelhecimento Cerebral; ESCADE, Escala de Avaliação de Capacidade de Decisão em Idosos; GDS, Escala de Depressão Geriátrica (Geriatric Depression Scale); HSL-PUCRS, Hospital São Lucas da PUCRS; PENCE, Programa de Envelhecimento Cerebral; TCLE, Termo de Consentimento Livre e Esclarecido.

\section{INTRODUÇÃO}

O envelhecimento populacional vem ocorrendo como fenômeno mundial. No Brasil, nas últimas décadas, houve um expressivo aumento do número de idosos, sendo que 2016, o Rio Grande do Sul era o estado que possuía a maior quantidade de idosos (16,19\%), seguido do Rio de Janeiro (15,13\%). De acordo com as projeções do Instituto Brasileiro de Geografia e Estatística, esse aumento tende a se ampliar, podendo alcançar no ano 2030 uma população total de $18,62 \%$ de idosos no Brasil [1]. Assim como ocorre com a expectativa no envelhecimento populacional, há projeções que apontam um crescimento de indivíduos portadores de transtornos mentais, que por sua vez podem acompanhar o envelhecimento humano [2]. Entre esses transtornos, a depressão enseja uma atenção especial, por ser frequentemente associada ao envelhecimento, apesar de muitas vezes ser negligenciada ou mal diagnosticada. Sua prevalência em idosos é de $4,8 \%$ a $14,6 \%$, sendo que nos idosos hospitalizados chega a $22,0 \%$ e, quando se avaliam sintomas depressivos em idosos na comunidade, as proporções atingem $6,4 \%$ a $59,3 \%$ [2].

Segundo a Organização Mundial da Saúde, no Relatório Mundial de Saúde apresentado em 2001, a depressão maior já era a principal causa de incapacitação em todo o mundo, ocupando o quarto lugar entre as principais causas de incapacitação por doença e passando a representar um problema de saúde pública [3]. Dentre os transtornos mentais que acometem os idosos, a depressão é a mais recorrente forma de sofrimento emocional e a que mais prejudica a qualidade de vida [4].

No contexto dessas duas realidades, surge um de seus reflexos: as demandas na esfera jurídica. Casos como pedidos de curatela para idosos são frequentes. Pelo processo de interdição, a incapacidade de uma pessoa será suprida pela sua representação por outra pessoa, designada como curador. Esses processos requerem o embasamento de laudos médicos, em especial de psiquiatras, pois é necessário avaliar o grau de autonomia do idoso e a sua capacidade de decisão.
Entre as causas de pedidos de interdição de idosos relacionadas a doenças, está presente a depressão [5].

$\mathrm{O}$ apoio de terceiros para quem necessita suprir a falta temporária, ou permanente, de autonomia, pode ser obtido atualmente por meio da Tomada de Decisão Apoiada, que ingressou no ordenamento jurídico brasileiro em janeiro de 2016 com o Estatuto da Pessoa com Deficiência, alterando a curatela no Código Civil [6]. Esse novo instituto jurídico revogou a antiga possibilidade de curatela dos enfermos ou deficientes mentais sem discernimento para os atos da vida civil. Com a Tomada de Decisão Apoiada, a pessoa com deficiência elegerá no mínimo duas pessoas idôneas, com as quais mantenha vínculo e em quem confie, para que lhe auxiliem nas suas decisões, permitindo, assim, que a pessoa com alguma deficiência exerça sua capacidade civil [7]. Essas pessoas não serão representantes ou assistentes, pois não haverá incapacidade civil, mas, sim, apoiadores. A pessoa com deficiência que está sendo apoiada manterá sua autonomia, não estando interditada ou incapacitada [8].

Além das alterações trazidas pelo Estatuto da Pessoa com Deficiência [7] em janeiro de 2016, em março de 2016 o ordenamento jurídico sofre novas mudanças com a entrada em vigor do novo Código de Processo Civil [9]. São modificações que buscam demonstrar a mudança conceitual, tanto nos aspectos psiquiátricos, quanto do ponto de vista social, ao que tange o entendimento da doença mental e sua inserção no ordenamento jurídico [10]. Ainda há a possibilidade da curatela para esses casos, porém é medida excepcional, usada apenas em casos de extrema necessidade.

A Tomada de Decisão Apoiada é mais flexível que a curatela e evita a estigmatização social do deficiente, que poderá manter sua autogestão, devendo sua vontade ser respeitada. Ela visa manter a dignidade da pessoa, estando mais voltada para o indivíduo que dela necessita do que a curatela, visto que, não raras as vezes, esta última volta-se mais para questões patrimoniais e de isolamento social. Ademais, ocorrerá a preservação do convívio familiar e com a comunidade [11].

Estudos que objetivem a avaliação mais precisa e específica dos momentos de alteração da autonomia mostram-se de suma importância para os idosos em situação de vulnerabilidade. A avaliação sobre a capacidade de decisão, especialmente, possibilita uma interferência correta, evitando possíveis abusos dos direitos dos idosos, respeitando de forma plena sua dignidade. Levando em conta as considerações acima, o objetivo do presente estudo foi verificar se 
há alterações na capacidade de decisão em idosos diagnosticados com depressão maior.

\section{MÉTODOS}

\section{Delineamento, local do estudo e ética}

Foi realizado um estudo de coorte com análise transversal inicial, utilizando um grupo de comparação, com coleta prospectiva de dados. O estudo foi conduzido no Ambulatório de Envelhecimento Cerebral (AMBEC) do Programa de Envelhecimento Cerebral (PENCE) desenvolvido como parceria entre o Hospital São Lucas da PUCRS (HSL-PUCRS) e a Secretaria Municipal de Saúde de Porto Alegre, no período de janeiro de 2014 a setembro de 2015. A pesquisa somente foi iniciada após a aprovação do Comitê de Ética em Pesquisa da Pontifícia Universidade Católica do Rio Grande do Sul, sob o no 24134013.0.0000.5336, e todos os participantes firmaram, em duas vias, o Termo de Consentimento Livre e Esclarecido (TCLE).

\section{Seleção da amostra e aplicação dos instrumentos de avaliação}

Os critérios de inclusão no grupo de estudo foram idade mínima de 60 anos, diagnóstico de episódio depressivo maior atual sem tratamento para o mesmo até o momento da inclusão, e aceite em realizar tratamento com a equipe de psiquiatras do AMBEC/ HSL-PUCRS. Foram excluídos da amostra idosos que apresentavam declínio cognitivo, outro transtorno psiquiátrico (que não a depressão maior atual) e/ou doença do sistema nervoso central.

Para avaliação inicial da presença de depressão foi utilizada a Escala de Depressão Geriátrica (GDS - Geriatric Depression Scale) na sua forma abreviada (GDS-15) [12]. Como ponto de corte para identificação de depressão, os idosos deveriam apresentar pontuação de GDS $\geq 5$.

Para identificar preliminarmente algum declínio cognitivo (critério de exclusão) foi usado o Instrumento de Triagem para Demência Vellore, traduzido e adaptado para uso no Brasil, que possui 10 questões para o paciente e 10 questões para um cuidador informante [13]. Para rastrear os idosos com declínio cognitivo, os pontos de corte utilizados no referido instrumento foram $\leq 11$ (paciente) e $\geq 5$ (informante).

Esses dois instrumentos foram aplicados por Agentes Comunitários de Saúde (ACE) treinados, em visita domiciliar, a todos os idosos cadastrados na Estratégia Saúde da Família de Porto Alegre nas regiões Leste, Lomba do Pinheiro/Partenon. De acordo com a parceria entre a Secretaria Municipal de Saúde e o HSL-PUCRS, todos os idosos eram encaminhados ao PENCE.

Além disso, os idosos identificados com depressão eram encaminhados pelos ACS para avaliação por psiquiatras e neurologistas no AMBEC/HSL-PUCRS. Para avaliar os critérios de inclusão e exclusão no estudo, testes adicionais foram aplicados nos idosos por esses especialistas.

O instrumento Mini International Neuropsychiatric Interview (MINI), na versão mais detalhada (MINIPlus), que lista diferentes categorias de transtornos mentais e critérios diagnósticos, sendo compatível com a versão IV do Manual Diagnóstico e Estatístico de Transtornos Mentais da Associação Americana de Psiquiatria [14], foi aplicado por psiquiatras para avaliar a presença de transtornos mentais e incluir no grupo de estudo apenas idosos que apresentassem critérios diagnósticos para depressão maior, na ausência de outros transtornos mentais. Ainda, o Exame Cognitivo de Addenbrooke - versão revisada [15] foi aplicado por neurologistas para identificar a presença de algum distúrbio cognitivo que também determinasse a exclusão do idoso da amostra selecionada. Se confirmada a presença de depressão maior sem tratamento, na ausência de outro transtorno mental, assim como de qualquer distúrbio cognitivo, os idosos eram convidados a participar da pesquisa e assinar o TCLE, sendo nesse momento preenchida a ficha com dados sociodemográficos daqueles que aceitaram.

O grupo controle foi composto por idosos cadastrados no PENCE sem sinais ou sintomas de depressão conforme o GDS (considerando normal com GDS $\leq 4$ ) e sem declínio cognitivo (considerando não alterado Vellore-paciente $\geq 12$ ou Vellore-informante $\leq 4)$. Por não terem diagnóstico de depressão ou doenças neurológicas, esses idosos não foram encaminhados para o AMBEC. Assim, a coleta de dados desse grupo ocorreu nos postos das Estratégias Saúde da Família, com agendamento de horário. Os que não puderam comparecer receberam visita domiciliar da pesquisadora, acompanhada pelos ACS, e foram convidados para a pesquisa. Daqueles que aceitaram participar e assinaram o TCLE, foram coletados os dados sociodemográficos.

\section{Avaliação da capacidade de decisão}

Para avaliação da capacidade de decisão dos idosos participantes (tanto do grupo de estudo como do grupo controle), foi aplicada a Escala de Avaliação 
de Capacidade de Decisão (ESCADE). Essa escala foi desenvolvida e validada pelo mesmo grupo de pesquisadores, em uma etapa anterior da pesquisa. Trata-se de um instrumento com perguntas e respostas baseadas na Escala Likert. A validação do instrumento foi feita pelo cálculo de alfa de Cronbach, em um total do alfa de 0,814 , mostrando confiabilidade e validade. O instrumento é composto por 18 questões e está dividido em quatro domínios: atividade diária, gestão financeira, autogestão e bem-estar [16].

A ESCADE foi aplicada, pela pesquisadora, a todos os idosos incluídos na pesquisa, e os resultados relativos à capacidade de decisão foram comparados entre o grupo de estudo (idosos com depressão maior) e o grupo controle.

$\mathrm{Na}$ seguinte etapa do estudo, o grupo de idosos com depressão maior foi submetido a tratamento psiquiátrico com a equipe de psiquiatras do PENCE/ HSL-PUCRS. O tratamento foi realizado por seis meses e consistiu em psicoterapia associada a tratamento medicamentoso. Após o tratamento, foi novamente aplicada a ESCADE. Os resultados da ESCADE pós tratamento foram comparados com os obtidos pelo mesmo grupo antes do tratamento.

\section{Processamento dos dados e análise estatística}

Após a coleta de dados, ocorreu uma seleção, no banco de dados, balanceando a amostra por sexo, idade e escolaridade. Para o cálculo do tamanho amostral foi utilizado um erro alfa de $5 \%$ e um poder de $80 \%$. O cálculo foi feito com base nos possíveis resultados obtidos com a ESCADE. Para comparar a média dos resultados do instrumento entre os grupos de idosos com e sem depressão, com um tamanho de efeito de 0,5 (diferença entre as médias de $50 \%$ do desvio padrão), em uma proporção de um caso para três controles, seriam necessários 42 idosos com depressão e 126 controles. Para comparar a modificação da média após o tratamento da depressão com tamanho de efeito de 0,4 (diferença de $40 \%$ do desvio padrão) seriam necessários 49 idosos.

Os dados foram digitados em planilha eletrônica Excel e analisados pelo programa estatístico SPSS versão 17.0 para Windows, por meio de estatística descritiva (frequências, médias e desvios-padrões) e analítica. $\mathrm{Na}$ análise transversal, foi comparado o grupo de estudo (idosos com episódio depressivo maior atual) com o grupo controle. Para comparação da média de idade foi utilizado o teste $t$ de Student para amostras independentes com variâncias iguais. Para comparação dos dados sociodemográficos categóricos, foi utilizado o teste do qui-quadrado de Pearson. Para comparação dos escores totais e dos domínios da ESCADE (atividade diária, gestão financeira, autogestão e bem-estar), além de cada questão, foi utilizado o teste de Mann-Whitney. Na análise longitudinal, para comparação entre os escores anteriores e posteriores ao tratamento, foi utilizado o teste de Wilcoxon. Foi considerado significativo um $\mathrm{p}<0,05$.

\section{RESULTADOS}

Foram estudados 48 idosos com diagnóstico de episódio depressivo maior atual, atendidos no AMBEC do HSL-PUCRS, e 144 controles (idosos da comunidade com GDS $\leq 4$ e sem distúrbios cognitivos). A idade variou de 60 a 85 anos, com média e desvio padrão de

Tabela 1. Dados sociodemográficos de idosos com depressão maior atual $(n=48)$ em comparação com o grupo controle, sem depressão $(n=144)$. Ambulatório de Envelhecimento Cerebral do Hospital São Lucas da PUCRS, Porto Alegre, RS, Brasil, 2014/2015.

\begin{tabular}{|c|c|c|c|}
\hline \multirow[t]{2}{*}{ Variável } & $\begin{array}{l}\text { Grupo com } \\
\text { depressão }\end{array}$ & $\begin{array}{l}\text { Grupo } \\
\text { controle }\end{array}$ & \multirow[t]{2}{*}{ p } \\
\hline & n (\%) & n (\%) & \\
\hline \multicolumn{4}{|l|}{ Sexo } \\
\hline Feminino & $42(87,5)$ & $122(84,7)$ & \multirow{2}{*}{$0,640^{*}$} \\
\hline Masculino & $6(12,5)$ & $22(15,3)$ & \\
\hline \multicolumn{4}{|l|}{ Escolaridade } \\
\hline $0-3$ & $16(33,3)$ & $36(25,0)$ & \multirow{3}{*}{$0,354^{+}$} \\
\hline $4-7$ & $16(33,3)$ & $44(30,6)$ & \\
\hline 8 ou + & $16(33,3)$ & $64(44,4)$ & \\
\hline \multicolumn{4}{|l|}{ Faixa etária } \\
\hline $60-69$ & $33(68,8)$ & $99(68,8)$ & \multirow{3}{*}{$0,886^{+}$} \\
\hline $70-79$ & $12(25,0)$ & $38(26,4)$ & \\
\hline $80 \mathrm{ou}+$ & $3(6,2)$ & $7(4,9)$ & \\
\hline \multicolumn{4}{|l|}{ Renda } \\
\hline Sem remuneração & $9(18,8)$ & $15(10,4)$ & \multirow{5}{*}{$0,161^{+}$} \\
\hline Até 1 salário mínimo & $23(47,9)$ & $71(49,3)$ & \\
\hline Até 2 salários mínimos & $11(22,9)$ & $37(25,7)$ & \\
\hline Até 3 salários mínimos & $4(8,3)$ & $13(9,0)$ & \\
\hline + de 4 salários mínimos & $1(2,1)$ & $8(5,6)$ & \\
\hline \multicolumn{4}{|l|}{ Cor } \\
\hline Branca & $31(64,6)$ & $87(60,4)$ & \multirow{4}{*}{$0,448^{*}$} \\
\hline Parda & $9(18,8)$ & $39(27,1)$ & \\
\hline Negra & $8(16,7)$ & $16(11,1)$ & \\
\hline Indígena & $0(0)$ & $2(1,4)$ & \\
\hline \multicolumn{4}{|l|}{ Estado civil } \\
\hline Solteiro & $4(8,3)$ & $17(11,8)$ & \multirow{4}{*}{$0,909^{*}$} \\
\hline Casado & $23(47,9)$ & $63(43,8)$ & \\
\hline Divorciado & $12(25)$ & $37(25,7)$ & \\
\hline Viúvo & $9(18,8)$ & $27(18,8)$ & \\
\hline
\end{tabular}

* Teste do qui-quadrado de Pearson.

† Teste de tendência linear do qui-quadrado. 
$67,6 \pm 7,0$ anos, no grupo de idosos com depressão; e de

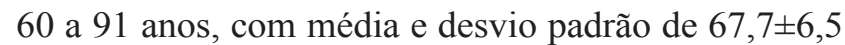
anos, no grupo controle $(\mathrm{p}=0,905)$. Houve a perda, por óbito, de uma idosa no transcorrer dos seis meses da coorte, a qual iria compor o número total estimado de 49 idosos no grupo de estudo. Os dados sociodemográficos comparando o grupo de idosos com depressão maior e o grupo controle são vistos na Tabela 1. Nessas variáveis não foi observada nenhuma diferença estatisticamente significativa entre os dois grupos.

Ao comparar a capacidade de decisão entre os idosos diagnosticados com depressão maior atual e o grupo controle, foi possível verificar que tanto no escore total da ESCADE quanto nos escores para os seus quatro domínios isoladamente, as diferenças foram significativas. A pontuação média da ESCADE em idosos deprimidos foi de $70,5 \pm 17,9$, enquanto

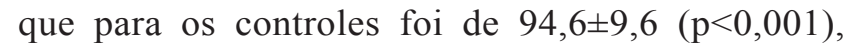
identificando menor capacidade de decisão nos idosos com depressão maior. Os domínios que apresentaram maior diferença foram o domínio 3 (autogestão) em que para os deprimidos a média foi de $65,0 \pm 23,3$ e para os controles foi de $97,8 \pm 6,2$, e o domínio 4 (bem-estar), em que a média nos deprimidos foi de 52,2 $\pm 27,1$, e nos controles de 91,8 116,7 (Tabela 2 ).

Tabela 2. Escores totais e em cada domínio da Escala de Avaliação de Capacidade de Decisão (ESCADE), obtidos pelos idosos com depressão maior $(n=48)$ em comparação com o grupo controle $(n=144)$. Ambulatório de Envelhecimento Cerebral do Hospital São Lucas da PUCRS, Porto Alegre, RS, Brasil, 2014/2015.

\begin{tabular}{|c|c|c|c|}
\hline \multirow[t]{2}{*}{ Variável } & $\begin{array}{l}\text { Grupo com } \\
\text { depressão }\end{array}$ & $\begin{array}{l}\text { Grupo } \\
\text { controle }\end{array}$ & \multirow[t]{2}{*}{$\mathbf{p}^{*}$} \\
\hline & Média $\pm D P$ & Média \pm DP & \\
\hline \multicolumn{4}{|l|}{ Domínios } \\
\hline 1. Atividade diária & $83,7 \pm 23,9$ & $92,2 \pm 22,6$ & $<0,001$ \\
\hline 2. Gestão financeira & $84,3 \pm 22,2$ & $94,0 \pm 16,7$ & $<0,001$ \\
\hline 3. Autogestão & $65,0 \pm 23,3$ & $97,8 \pm 6,2$ & $<0,001$ \\
\hline 4. Bem-estar & $52,2 \pm 27,1$ & $91,8 \pm 16,7$ & $<0,001$ \\
\hline Total & $70,5 \pm 17,9$ & $94,6 \pm 9,6$ & $<0,001$ \\
\hline
\end{tabular}

DP, desvio padrão.

* Teste de Mann-Whitney.

Tabela 3. Comparação entre o grupo de idosos com diagnóstico de depressão maior $(n=48)$ e o grupo controle $(n=144)$ por cada questão da Escala de Avaliação de Capacidade de Decisão (ESCADE). Ambulatório de Envelhecimento Cerebral do Hospital São Lucas da PUCRS, Porto Alegre, RS, Brasil, 2014/2015.

\begin{tabular}{|c|c|c|c|}
\hline \multirow{2}{*}{ Variável } & Grupo com depressão & Grupo controle & \multirow{2}{*}{$\mathbf{p}^{*}$} \\
\hline & Média \pm DP & Média \pm DP & \\
\hline \multicolumn{4}{|l|}{ Domínio 1 (Atividade diária) } \\
\hline $\mathrm{O}(\mathrm{A}) \operatorname{Sr}(\mathrm{a})$. toma banho sozinho(a)? & $5,00 \pm 0,00$ & $4,83 \pm 0,80$ & 0,152 \\
\hline O(A) Sr(a). arruma a casa sozinho(a)? & $4,17 \pm 1,33$ & $4,69 \pm 1,04$ & $<0,001$ \\
\hline $\mathrm{O}(\mathrm{A}) \mathrm{Sr}(\mathrm{a})$. cozinha? & $4,04 \pm 1,56$ & $4,56 \pm 1,16$ & 0,008 \\
\hline O(A) Sr(a). auxilia nas tarefas domésticas? & $4,19 \pm 1,39$ & $4,67 \pm 1,08$ & 0,001 \\
\hline \multicolumn{4}{|l|}{ Domínio 2 (Gestão financeira) } \\
\hline $\mathrm{O}$ (A) Sr(a). escolhe o que faz com seu dinheiro? & $4,81 \pm 1,14$ & $5,02 \pm 0,66$ & 0,588 \\
\hline O(A) Sr(a). administra o seu dinheiro? & $4,83 \pm 1,23$ & $4,97 \pm 0,81$ & 0,788 \\
\hline $\mathrm{O}(\mathrm{A}) \mathrm{Sr}(\mathrm{a})$. administra as contas da casa? & $3,71 \pm 1,90$ & $4,49 \pm 1,33$ & 0,005 \\
\hline O(A) Sr(a). escolhe o que come? & $4,92 \pm 0,58$ & $4,99 \pm 0,08$ & 0,408 \\
\hline \multicolumn{4}{|l|}{ Domínio 3 (Autogestão) } \\
\hline $\mathrm{O}(\mathrm{A}) \mathrm{Sr}(\mathrm{a})$. se sente apto(a) a tomar decisões? & $3,65 \pm 1,52$ & $4,94 \pm 0,31$ & $<0,001$ \\
\hline $\mathrm{O}(\mathrm{A}) \operatorname{Sr}(\mathrm{a})$. toma decisões sobre a sua vida? & $3,73 \pm 1,55$ & $4,94 \pm 0,35$ & $<0,001$ \\
\hline O(A) Sr(a). já se sentiu desinteressado(a) pela sua vida? & $2,50 \pm 1,53$ & $4,90 \pm 0,51$ & $<0,001$ \\
\hline O(A) Sr(a). costuma ir sozinho(a) aos supermercado/farmácia/banco? & $3,31 \pm 1,88$ & $4,78 \pm 0,86$ & $<0,001$ \\
\hline O(A) Sr(a). tem liberdade de visitar os seus amigos? & $4,33 \pm 1,43$ & $4,97 \pm 0,23$ & $<0,001$ \\
\hline $\mathrm{O}(\mathrm{A}) \operatorname{Sr}(\mathrm{a})$. administra sua vida com autonomia? & $4,08 \pm 1,43$ & $4,94 \pm 0,41$ & $<0,001$ \\
\hline \multicolumn{4}{|l|}{ Domínio 4 (Bem-estar) } \\
\hline $\mathrm{O}(\mathrm{A}) \mathrm{Sr}(\mathrm{a})$. se sente feliz com a sua vida? & $2,21 \pm 1,20$ & $4,83 \pm 0,56$ & $<0,001$ \\
\hline $\mathrm{O}(\mathrm{A}) \mathrm{Sr}(\mathrm{a})$. costuma sair sozinho para passear? & $2,77 \pm 1,90$ & $4,63 \pm 1,11$ & $<0,001$ \\
\hline $\mathrm{O}(\mathrm{A}) \mathrm{Sr}(\mathrm{a})$. tem liberdade de receber seus amigos em casa? & $4,54 \pm 1,20$ & $4,97 \pm 0,33$ & $<0,001$ \\
\hline O(A) Sr(a). pode viajar sozinho? & $2,83 \pm 2,01$ & $4,26 \pm 1,54$ & $<0,001$ \\
\hline
\end{tabular}

DP, desvio padrão.

* Teste de Mann-Whitney. 
Foram comparados os idosos do grupo de estudo (com depressão maior atual) com os do grupo controle, tendo por variáveis as questões da ESCADE que se dividem em domínios. No domínio 1 (atividade diária), apenas o questionamento referente ao banho não foi significativamente diferente entre os dois grupos. No domínio 2 (gestão financeira) apenas o questionamento relacionado com a administração das contas da casa teve diferença significativa $(\mathrm{p}=0,005)$. Já os domínios 3 (autogestão) e 4 (bem-estar), mostraram significância estatística em todas as perguntas elaboradas (Tabela 3).

Tanto para a pontuação geral quanto para cada um dos quatro domínios da ESCADE, foi significativa a diferença entre a capacidade de tomada de decisão antes e após o tratamento psiquiátrico dos idosos com depressão: a capacidade de decisão foi maior após o tratamento (Tabela 4).

Quando comparados os idosos com depressão maior atual antes e após o tratamento, observou-se que no domínio 1 (atividade diária) apenas a questão que se referia às tarefas domésticas apresentou diferença significativa $(\mathrm{p}=0,027)$. No domínio 2 (gestão financeira) nenhuma questão teve diferença signi- ficativa, enquanto que no domínio 3 (autogestão) todas as questões foram significativamente diferentes. Já no domínio 4 (bem-estar) apenas uma pergunta não teve diferença significativa: a que se refere a ter liberdade de receber os amigos em casa (Tabela 5).

Tabela 4. Escores totais e em cada domínio da Escala de Avaliação de Capacidade de Decisão (ESCADE), no grupo de idosos com depressão maior atual $(n=48)$, antes e após o tratamento psiquiátrico. Ambulatório de Envelhecimento Cerebral do Hospital São Lucas da PUCRS, Porto Alegre, RS, Brasil, 2014/2015.

\begin{tabular}{lcccc}
\hline Variável & $\begin{array}{c}\text { Antes do } \\
\text { tratamento } \\
\text { psiquiátrico }\end{array}$ & & $\begin{array}{c}\text { Após o } \\
\text { tratamento } \\
\text { psiquiátrico }\end{array}$ & \multirow{2}{*}{$\mathbf{p}^{*}$} \\
\cline { 2 - 2 } Média \pm DP & & Média \pm DP & \\
Domínios & & & \\
1. Atividade diária & $83,7 \pm 23,9$ & & $93,0 \pm 18,2$ & $\mathbf{0 , 0 4 6}$ \\
2. Gestão financeira & $84,3 \pm 22,2$ & & $92,0 \pm 17,7$ & $\mathbf{0 , 0 4 5}$ \\
3. Autogestão & $65,0 \pm 23,3$ & & $88,9 \pm 14,5$ & $<\mathbf{0 , 0 0 1}$ \\
4. Bem-estar & $52,2 \pm 27,1$ & & $80,8 \pm 23,6$ & $<\mathbf{0 , 0 0 1}$ \\
Total & $70,5 \pm 17,9$ & $88,7 \pm 14,7$ & $<\mathbf{0 , 0 0 1}$ \\
\hline
\end{tabular}

DP, desvio padrão.

* Teste de Wilcoxon.

Tabela 5. Escores obtidos em cada questão da Escala de Avaliação de Capacidade de Decisão (ESCADE), antes e após o tratamento dos idosos para depressão maior atual $(n=48)$. Ambulatório de Envelhecimento Cerebral do Hospital São Lucas da PUCRS, Porto Alegre, RS, Brasil, 2014/2015.

\begin{tabular}{|c|c|c|c|}
\hline \multirow[t]{2}{*}{ Questões } & $\begin{array}{l}\text { Antes do tratamento } \\
\text { psiquiátrico }\end{array}$ & $\begin{array}{l}\text { Após o tratamento } \\
\text { psiquiátrico }\end{array}$ & \multirow[t]{2}{*}{$\mathbf{p}^{*}$} \\
\hline & $\mathrm{M} \pm \mathrm{DP}$ & $\mathrm{M} \pm \mathrm{DP}$ & \\
\hline \multicolumn{4}{|l|}{ Domínio 1 (Atividade diária) } \\
\hline $\mathrm{O}(\mathrm{A}) \mathrm{Sr}(\mathrm{a})$. toma banho? sozinho(a) & $5,00 \pm 0,00$ & $4,96 \pm 0,29$ & 0,317 \\
\hline $\mathrm{O}(\mathrm{A}) \operatorname{Sr}(\mathrm{a})$. arruma a casa sozinho(a)? & $4,17 \pm 1,33$ & $4,63 \pm 1,06$ & 0,080 \\
\hline $\mathrm{O}(\mathrm{A}) \mathrm{Sr}(\mathrm{a})$. cozinha? & $4,04 \pm 1,56$ & $4,58 \pm 1,16$ & 0,078 \\
\hline $\mathrm{O}(\mathrm{A}) \mathrm{Sr}(\mathrm{a})$. auxilia nas tarefas domésticas? & $4,19 \pm 1,39$ & $4,71 \pm 0,92$ & 0,027 \\
\hline \multicolumn{4}{|l|}{ Domínio 2 (Gestão financeira) } \\
\hline $\mathrm{O}$ (A) Sr(a). escolhe o que faz com seu dinheiro? & $4,81 \pm 1,14$ & $4,98 \pm 0,67$ & 0,408 \\
\hline $\mathrm{O}(\mathrm{A}) \mathrm{Sr}(\mathrm{a})$. administra o seu dinheiro? & $4,83 \pm 1,23$ & $4,75 \pm 1,04$ & 0,524 \\
\hline $\mathrm{O}(\mathrm{A}) \mathrm{Sr}(\mathrm{a})$. administra as contas da casa? & $3,71 \pm 1,90$ & $4,29 \pm 1,50$ & 0,060 \\
\hline O(A) Sr(a). escolhe o que come? & $4,92 \pm 0,58$ & $4,96 \pm 0,29$ & 0,655 \\
\hline \multicolumn{4}{|l|}{ Domínio 3 (Autogestão) } \\
\hline $\mathrm{O}(\mathrm{A}) \mathrm{Sr}(\mathrm{a})$. se sente apto(a) a tomar decisões? & $3,65 \pm 1,52$ & $4,73 \pm 0,89$ & 0,001 \\
\hline $\mathrm{O}(\mathrm{A}) \operatorname{Sr}(\mathrm{a})$. toma decisões sobre a sua vida & $3,73 \pm 1,55$ & $4,73 \pm 0,89$ & 0,001 \\
\hline $\mathrm{O}(\mathrm{A}) \operatorname{Sr}(\mathrm{a})$. já se sentiu desinteressado(a) pela sua vida? & $2,50 \pm 1,53$ & $3,60 \pm 1,50$ & 0,001 \\
\hline $\mathrm{O}(\mathrm{A}) \mathrm{Sr}(\mathrm{a})$. costuma ir sozinho(a) aos supermercado/farmácia/banco? & $3,31 \pm 1,88$ & $4,58 \pm 1,18$ & $<0,001$ \\
\hline O(A) Sr(a). tem liberdade de visitar os seus amigos? & $4,33 \pm 1,43$ & $4,75 \pm 0,98$ & 0,025 \\
\hline O(A) Sr(a). administra sua vida com autonomia? & $4,08 \pm 1,43$ & $4,94 \pm 0,24$ & $<0,001$ \\
\hline \multicolumn{4}{|l|}{ Domínio 4 (Bem-estar) } \\
\hline $\mathrm{O}(\mathrm{A}) \mathrm{Sr}(\mathrm{a})$. se sente feliz com a sua vida? & $2,21 \pm 1,20$ & $3,92 \pm 1,05$ & $<0,001$ \\
\hline $\mathrm{O}(\mathrm{A}) \mathrm{Sr}(\mathrm{a})$. costuma sair sozinho para passear? & $2,77 \pm 1,90$ & $4,42 \pm 1,27$ & $<0,001$ \\
\hline $\mathrm{O}(\mathrm{A}) \mathrm{Sr}(\mathrm{a})$. tem liberdade de receber seus amigos em casa? & $4,54 \pm 1,20$ & $4,83 \pm 0,81$ & 0,245 \\
\hline $\mathrm{O}(\mathrm{A}) \mathrm{Sr}(\mathrm{a})$. pode viajar sozinho? & $2,83 \pm 2,01$ & $3,75 \pm 1,87$ & 0,012 \\
\hline
\end{tabular}




\section{DISCUSSÃO}

No presente estudo foi identificada menor capacidade de decisão em idosos com depressão maior atual quando comparados a um grupo controle sem depressão. A alteração de capacidade de tomada de decisão já havia sido evidenciada na presença de doenças neurológicas, mesmo naquelas que causam estados leves de demência [17], levando ao comprometimento das ações da vida diária. Entretanto, ao nosso conhecimento, a associação entre depressão maior e alteração na capacidade de decisão, na ausência de outros transtornos mentais ou déficit cognitivo, é um achado inédito.

Estudos realizados com idosos deprimidos não costumam dar o enfoque da autonomia civil aqui abordada, com ênfase para os limites legais da curatela. As pesquisas publicadas mostram geralmente a incapacidade funcional no âmbito das atividades de vida diária ou da qualidade de vida, ou ainda, enfocam algum ponto específico, como as questões econômicas. Dessa forma, buscou-se unir três importantes temas: idosos, depressão e autonomia civil.

Uma pesquisa publicada em 2016 avaliou a capacidade de tomada de decisão em idosos institucionalizados e não institucionalizados. $\mathrm{O}$ trabalho não apontou doenças relacionadas aos idosos ou depressão, como o presente estudo, mas apenas o fato da institucionalização. Os autores puderam identificar, em uma amostra de idosos de uma cidade do interior do Rio Grande do Sul, que todos os participantes tinham capacidade para tomar decisões, reconhecendo-os como "sujeitos da própria vontade" [18].

Com relação aos dados sociodemográficos, no presente estudo as amostras do grupo de estudo e do grupo controle foram compostas por idosos com proporção semelhante de escolaridade, sexo e idade. Além disso, ambos os grupos faziam parte da mesma região da cidade, vindos da Estratégia Saúde da Família. Assim, não foi possível identificar diferenças entre os idosos com ou sem depressão, decorrentes dos dados sociodemográficos, que pudessem interferir nos resultados encontrados.

Os domínios relacionados com autogestão e bemestar foram os que mostraram maior variação entre idosos deprimidos e não deprimidos. Justamente no domínio autogestão encontram-se questões como sentir-se apto a tomar decisões e realmente decidir sobre a sua vida. O domínio bem-estar, que inclui o sentimento de felicidade e a vontade de interação com o mundo, apresenta maior relação com as alterações decorrentes da doença em estudo, a depressão.
As alterações mais acentuadas encontradas nos domínios acima descritos fundamentam a Tomada de Decisão Apoiada. Com o apoiador para as pessoas com doenças mentais (que incluem a depressão maior) não haverá a supressão total da capacidade do indivíduo, mas o auxílio específico para as suas necessidades, em determinado momento comprometidas. Dessa forma, não haverá privação de direitos [8].

Como mostram os dados da Tabela 3, que discrimina as respostas às questões de cada domínio, o domínio 2, que diz respeito à gestão financeira, é o que contém respostas com menor alteração nos idosos deprimidos. Os dados indicam uma preservação da capacidade de decidir o que deseja comer e da capacidade para gerir seu próprio dinheiro, mas não as contas da casa. Souza et al. [19] investigaram instrumentos capazes de verificar a alteração em consentir financeiramente, concluindo pela necessidade do profissional da saúde em estar apto para a avaliação mais ampla do estado de saúde do paciente e incluindo as questões financeiras, permitindo uma melhor interpretação do diagnóstico, em especial ao que condiz com as questões legais. Os demais domínios mostram as alterações que ocorrem na depressão maior, enfatizando a importância da utilização de instrumentos capazes de auxiliar o diagnóstico mais preciso da perda da capacidade de decisão e da área específica em que a mesma ocorreu. Neste sentido, um estudo apresentado em 2015 concluiu que no contexto em que vivemos, com o envelhecimento populacional e o aumento na prevalência de doenças relacionadas a essa faixa etária, os profissionais, tanto da saúde quando da área social, precisam estar equipados com instrumentos para proteção dos direitos da autonomia dos vulneráveis [20].

A necessidade de avaliação da capacidade de decisão dos idosos também foi objeto de um estudo realizado na Nova Zelândia, o qual identificou que de 1347 idosos internados, apenas 87 tinham sido avaliados quanto à sua capacidade de decisão. Os autores concluíram pela importância do treinamento de enfermeiros e clínicos gerais para aplicação de instrumento capaz de realizar a verificação da capacidade de consentir dos pacientes [21].

O presente estudo, ao comparar os idosos deprimidos antes e após o tratamento psiquiátrico, amplia o entendimento sobre a capacidade de decisão dos idosos, uma vez que mostra a alteração total pela escala ESCADE, bem como em cada um dos seus quatro domínios. Faz-se, portanto, possível a averiguação exata da perda, devendo-se ressaltar a identificação das questões que sofreram mudanças significativas após o tratamento. A convergência aqui 
enfatizada, entre o diagnóstico médico e seus reflexos na esfera jurídica, é importante diante da sociedade hodierna, permitindo uma avaliação mais precisa da capacidade de decisão e, assim, restrição apenas nas esferas necessárias [22].

Pode-se considerar como uma limitação deste estudo a falta de acompanhamento por meio de uma escala de adesão medicamentosa, que possibilitaria comprovar se os idosos em tratamento para depressão maior estavam fazendo uso correto da medicação indicada pela equipe de psiquiatras. Esse aspecto é importante, uma vez que a melhora desse transtorno é em grande parte atribuída ao uso de medicação específica. Cabe ressaltar, ainda, que poderia ter ocorrido uma aplicação da ESCADE em tempo intermediário (três meses), para verificar se a mudança na capacidade de decisão ocorreu antes do tempo total da pesquisa (seis meses).

Apesar dessas limitações, pode-se concluir que a aplicação da ESCADE identificou menor capacidade de decisão nos idosos no momento em que foram diagnosticados com depressão maior atual, havendo melhora após seis meses de tratamento psiquiátrico.
Considera-se pertinente estender o uso da ESCADE para outras doenças psiquiátricas, visando identificar aquelas que podem afetar a capacidade de decisão. Está-se diante de um importante meio auxiliar nas perícias, tanto para o uso dos psiquiatras, quanto para outras áreas médicas que necessitem de auxílio para comprovação em laudos, chegando ao uso dos magistrados. A medida complementar de diagnóstico pode embasar e respaldar o fundamento para questões como a curatela ou a Tomada de Decisão Apoiada.

\section{NOTAS}

\section{Agradecimentos}

Os autores agradecem à CAPES pela bolsa de doutorado e à Dra. Paula Engroff pela revisão do manuscrito.

\section{Apoio financeiro}

O estudo recebeu apoio financeiro da Coordenação de Aperfeiçoamento de Pessoal de Nível Superior (CAPES).

\section{Declaração de conflito de interesses}

Os autores declaram não haver conflitos de interesses relevantes ao conteúdo deste estudo, informam ter tido acesso a todos os dados obtidos e assumem completa responsabilidade pela integridade dos resultados.

\section{REFERÊNCIAS}

1. Brasil. Instituto Brasileiro de Geografia e Estatística. Projeção da população do Brasil e das Unidades da Federação [Internet]. 2016 [citado 13 de novembro de 2016]. p. 2. Available from: http://www.ibge.gov.br/apps/populacao/projecao/

2. Frank MH, Rodrigues NL. Depressão, Ansiedade, Outros Transtornos Afetivos e Suicídio. In: Freitas EV, Py L, organizadores. Tratado de Geriatria e Gerontologia. 3a ed. Rio de Janeiro: Guanabara Koogan, 2011. p. 314-26.

3. Organização Mundial de Saúde. Saúde mental: nova concepção, nova esperança [Internet]. 1aed. The World Health Report. Lisboa; 2002. Available from: http://apps.who.int/iris/bitstream/10665/42390/4/WHR_2001_por.pdf

4. Blazer DG. Depressão. In: Abrams WB, Berkow R, organizadores. Manual Merck de Geriatria. São Paulo: Roca; 1994. p. 1086-96.

5. Crippa A, Gomes I. Pedidos judiciais relacionados à interdição de idosos. Rev Kairós Gerontol. 2014;17(3):329-42. https://revistas.pucsp.br/index.php/kairos/article/view/23870

6. Brasil. Lei no 10.406 de 10 de janeiro de 2002. Institui o Código Civil. Brasília, 10 de janeiro de 2002.

7. Brasil. Lei no 13.146, de 6 de julho de 2015. Institui a Lei Brasileira de Inclusão da Pessoa com Deficiência (Estatuto da Pessoa com Deficiência). Brasília, 2015.

8. Farias CC, Rosenvald N. Curso de Direito Civil: famílias. 8aㅡ ed. Salvador: JusPodivm, 2016.

9. Brasil. Lei no 13.105, de 16 de março de 2015. Institui o Código de Processo Civil. Brasília, 2015.

10. Medeiros MBM. Interdição civil: proteção ou exclusão. São Paulo: Cortez, 2007.

11. Amorim AMA. Manual de direitos das famílias. Curitiba: Juruá, 2016.

12. Yesavage JA, Brink TL, Rose TL, Lum O, Huang V, Adey M, Leirer VO. Development and validation of a geriatric depression screening scale: a preliminary report. J Psychiatr Res. 1983;17(1):37-49. https://doi.org/10.1016/00223956(82)90033-4

13. Loureiro F, Finger G, Gomes I. Tradução e adaptação cultural para o português brasileiro do instrumento para rastreio cognitivo Vellore. PAJAR - Pan Am J Aging Res. 2015;3(2):47-52. https://doi.org/10.15448/2357-9641.2015.2.22950

14. Amorin P. Mini International Neuropsychiatric Interview (MINI): validação de entrevista breve para diagnóstico de transtornos mentais. Rev Bras Psiquiatr. 2000;22(3):106-15. https://doi.org/10.1590/S1516-44462000000300003

15. Mioshi E, Dawson K, Mitchell J, Arnold R, Hodges JR. The Addenbrooke's Cognitive Examination Revised (ACE-R): a brief cognitive test battery for dementia screening. Int J Geriatr Psychiatry. 2006;21(11):1078-85. https://doi.org/ 10.1002/gps. 1610 
16. Silva AC. Autonomia do idoso: avaliação da capacidade da tomada de decisão e associação com depressão maior [thesis]. [Porto Alegre]: Pontifícia Universidade Católica do Rio Grande do Sul; 2017. http://hdl.handle.net/10923/10049

17. Álvaro LC. Competencia: conceptos generales y aplicación en la demencia. Neurología. 2012;27(5):290-300. https://doi. org/10.1016/j.nrl.2011.12.005

18. Deon RG, Goldim JR. Capacidade para tomada de decisão em idosos institucionalizados e não institucionalizados. Estud Interdiscipl Envelhec. 2016;21(1):123-33.

19. Sousa LB, Simões MR, Firmino H, Peisah C. Financial and testamentary capacity evaluations: procedures and assessment instruments underneath a functional approach. Int Psychogeriatrics. 2014;26(2):217-28. https://doi.org/10.1017/ S1041610213001828

20. Giroux D, Tétreault S, Landry M-P. Evaluating adult's competency: application of the competency assessment process. Int J Alzheimers Dis. 2015;2015:1-7. https://doi.org/10.1155/2015/753873

21. Astell H, Lee JH, Sankaran S. Review of capacity assessments and recommendations for examining capacity. N Z Med J. 2013;126(1383):38-48.

22. Moye J, Marson DC. Assessment of Decision-Making Capacity in Older Adults: An Emerging Area of Practice and Research. J Gerontol B Psychol Sci Soc Sci. 2007;62(1):3-11. https://doi.org/10.1093/geronb/62.1.P3 